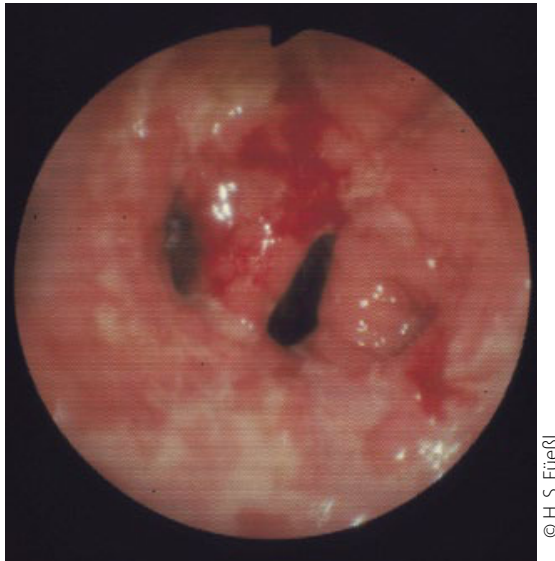

Die vollständige Mukosaheilung ist ein wichtiges Therapieziel bei Morbus Crohn.

Vor kurzem erhielt Infliximab zudem vom Ausschuss für Humanarzneimittel (CHMP) der Europäischen Arzneimittelagentur eine positive Stellungnahme für die Verkürzung der Infusionsdauer auf eine Stunde bei erwachsenen Patienten aller Indikationen, die drei initiale Infusionen vertragen haben.

- Red

Quelle: Nach Informationen von MSD

Bluthochdrucktherapie mit Fixkombination Effektive Zielwerteinstellung, reduzierte Ödemneigung

- Die Behandlungserfolge bei arterieller Hypertonie sind nach Prof. Thomas Unger, Berlin, immer noch suboptimal. Trotz einer breiten Palette von Antihypertonika werden die Zielwerte von $<140 / 90 \mathrm{mmHg}$ oft nicht erreicht. Nur die Hälfte aller Hypertoniker wird optimal kontrolliert.

Der unzureichenden RR-Kontrolle liegen verschiedene Ursachen zugrunde: Häufig werden Antihypertensiva in inadäquaten Dosierungen verschrieben, das Potenzial von Arzneimittel-Kombinationen wird nicht ausgeschöpft und die Compliance ist - v. a. bei Einnahme mehrerer Antihypertensiva unzureichend. Zwei Drittel der Patienten benötigen mindestens zwei blutdrucksenkende Wirkstoffe. Mit jeder zusätzlichen Tablette sinkt die Compliance. Zur Vereinfachung der Therapie und Verbesserung der Compliance empfiehlt die European Society of Hypertension (ESH), in der Bluthochdrucktherapie bevorzugt Fixkombinationen einzusetzen.

Wenn die Standardtherapie versagt

\title{
Auch Mariendistelextrakt kann Viruslast bei chronischer Hepatitis $C$ senken
}

- Ein Nichtansprechen auf eine Standardtherapie der Hepatitis C mit pegyliertem Interferon und Ribavirin (peg-INF/RBV) ist v. a. bei dem am meisten verbreiteten Genotyp 1 sehr häufig. Mit Silibinin (Legalon ${ }^{\oplus}$ SIL) steht ein wirksamer Therapieansatz für Therapieversager zur Verfügung, berichtete Prof. Thomas Berg, Leipzig. Die Therapie ist seit Anfang des Jahres in Österreich als Begleitmedikation bei Patienten mit chronischer Hepatitis $C$, die nicht oder ungenügend auf die antivirale Standardtherapie ansprechen, zugelassen.

Silibinin hemmt die Replikation der Hepatitis-C-Viren (HCV) durch eine direkte Inhibition der RNA-abhängigen RNA-Polymerase. Auf den oralen Mariendistelextrakt sind die positiven Ergebnisse nicht übertragbar, betonte Berg.
Eine klinische Studie mit peg-INF/RBVNon-Respondern hat gezeigt, dass eine hoch dosierte intravenöse Verabreichung von Silibinin die Viruslast dosisabhängig reduziert (Ferrenci $P$ et al. Gastroenterology 2008; 1561-67). Über einen Zeitraum von zwei bis drei Wochen erhielten die Studienteilnehmer tägliche Infusionen mit 15-20 $\mathrm{mg} / \mathrm{kg}$ KG Silibinin. Nach einer Woche Silibilin-Monotherapie wurden sie zusätzlich wieder mit peg-INF/RBV für 48 Wochen behandelt. Fast $90 \%$ der Patienten erreicht eine Reduktion der Viruslast um mindestens zwei Zehnerpotenzen. Und auch nach 25 Wochen war noch die Hälfte von ihnen virusfrei.

Die Daten einer neueren Studie zeigen, dass eine zweitägige Hochdosistherapie mit Silibinin ( $1400 \mathrm{mg} / \mathrm{d}$ ) bei 20 Patienten mit partieller Response auf peg-INF/RBV
Die Fixkombination aus dem Angiotensinrezeptorblocker Telmisartan und dem Kalziumantagonisten Amlodipin (Twynsta ${ }^{\circledR}$ ) bietet den Vorteil komplementärer und synergistischer Wirkmechanismen auf das Renin-Angiotensin-System (RAS) und die Blutgefäße, so Unger. Dies führt zu einer Reduktion des systolischen Blutdrucks um bis zu $50 \mathrm{mmHg}$. Zudem gleicht Telmisartan die durch den Kalziumantagonisten ausgelöste arterielle Vasodilatation durch eine zusätzliche venöse Dilatation aus, was gegenüber einer Amlodipin-Monotherapie die Ödemrate um 40-50\% reduziert. Telmisartan weist laut Unger eine gute Datenlage auf. Die ONTARGET-Studie zeigte, dass das Sartan bei Hochrisikopatienten genauso wirksam und besser verträglich ist als der ACE-Hemmer Ramipril.

- Abdol A. Ameri

Quelle:Twynsta Media Dinner: Aktuelle Hypertonietherapie, 117. Internistenkongress, Wiesbaden (Veranstalter: Boehringer Ingelheim) ein vollständiges und lang anhaltendes virologisches Ansprechen bewirkte (Biermer $M$ et al. AASLD 2010; \#989). Während der folgenden Woche sank die Viruslast unter fortgesetzter Standardtherapie bei 13 Patienten (65\%) unter die Nachweisgrenze.

Laut Berg wurden mit Silibinin auch schon erste Erfolge bei HIV/HCV-Koinfektion erzielt sowie bei Patienten, die nach einer Lebertransplantation eine HCV-Reinfektion erlitten hatten. Diese Daten deuten darauf hin, dass der Distelextrakt eine Therapiealternative für besonders schwer behandelbare HCV-Patienten darstellen könnte.

- Abdol A. Ameri

Quelle: Satellitensymposium „Silymarin/Silibinin: Updates on the medical management of HCV and NASH“, 46. EASL-Kongresses, Berlin, April 2011 (Veranstalter: Rottapharm Madaus) 